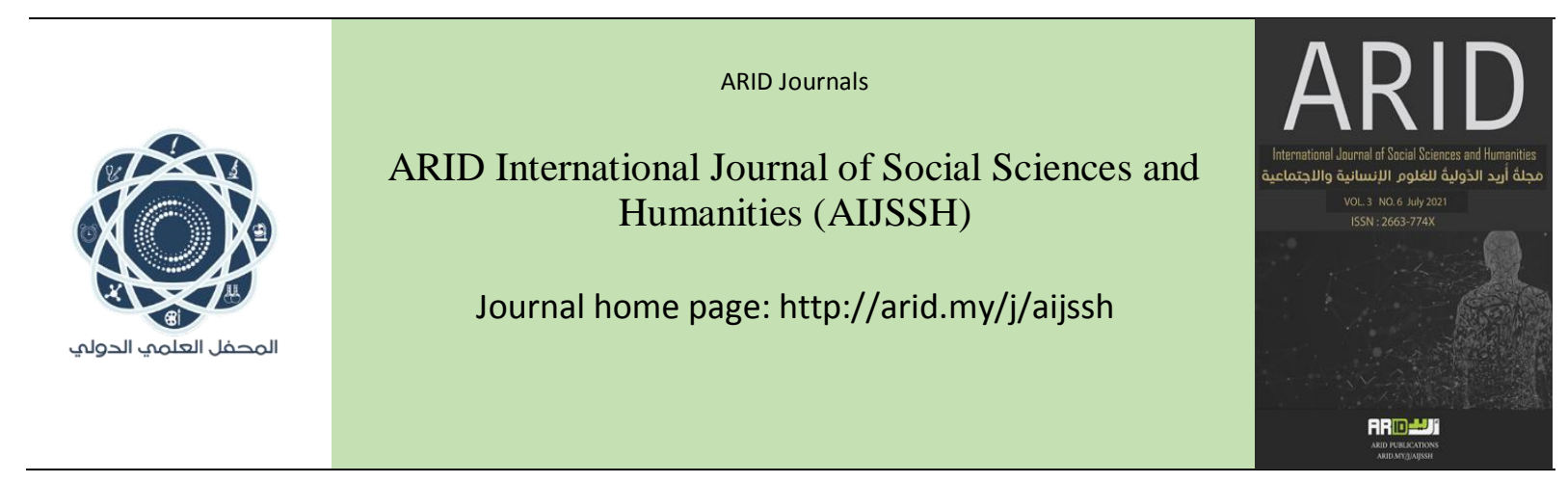

$$
\begin{aligned}
& \text { مَجلةُ أُريد الدَّوليُُّ للعُلومِ الإنسانية والإجتماعية } \\
& \text { البجلد الثالث ،العدد السادس، تموز } 2021 \text { م }
\end{aligned}
$$

\title{
Electronic classroom activities in Elementary schools (reality and challenges)
}

\author{
Azhar abdalbar \\ Faculty of Education - Zagazig University - Egypt \\ الأنشطة الصفية الإكترونية بالمدارس الأساسية \\ (الواقع والتحديات) \\ أزهار محمد محمد عبد البر \\ كلية التربية-جامعة الزقازيقهصر \\ azhar_abdalbar@yahoo.con \\ https://doi.org/10.36772/arid.aijssh.2021.364
}




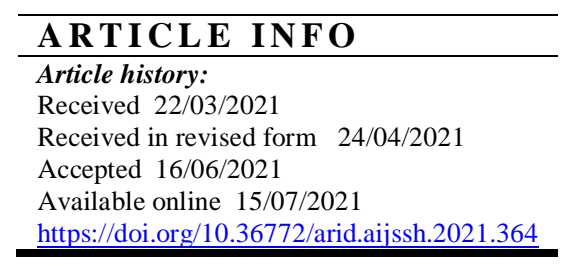

\begin{abstract}
The aim of the research is to identify the reality of the application of electronic classroom activities in Elementary schools, to identify the challenges that face the application of electronic classroom activities in primary schools, The research sample consisted of (120) male and female teachers from the basic education stage, (50) guardians, (15) school principals, (30) activity supervisors And the descriptive and analytical approach was used and a questionnaire on the application of electronic activities was applied (prepared by the researcher). The research found the following results: Elementary schools do not implement electronic classroom activities sufficiently, and this is due to many challenges, which are weak economic potential in primary schools, lack of infrastructure and adequate high-quality computers to implement electronic activities in schools, weak Internet and lack of Internet networks in many schools, teachers 'lack of experience in preparing and designing electronic classroom activities, poor economic conditions for students, especially in villages and rural areas where there is no infrastructure.
\end{abstract}

Keywords: Electronic classroom activities, Elementary schools ,reality , challenges. 


\section{الملخص}

هدف البحث التعرف على واقع تطبيق الأنشطة الإلكترونية الصفية في المدارس الأساسية، التعرف على التحدية التحديات التي تواجهه

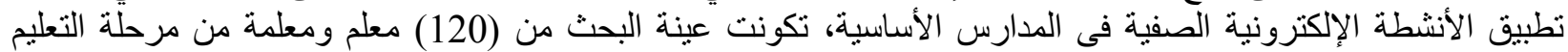

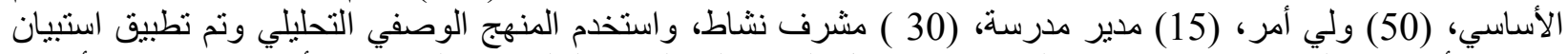

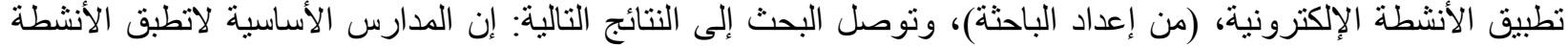

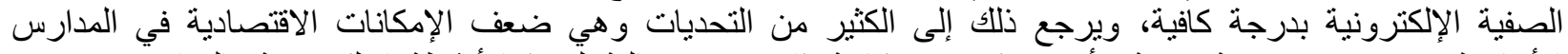



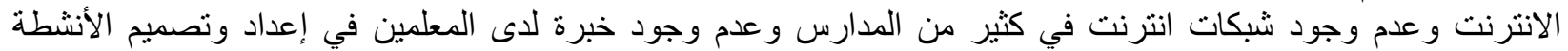

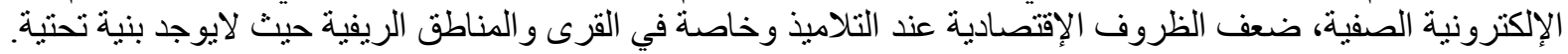
•الكلمات المفتاحية: الأنشطة الإكترونية_ المدارس الأساسية ـ الواقعـ التحديات . 
منلما اجتاح فيروس كورونا المستجد حواجز الزمان و المكان جاءت دعوات التعليم عن بعد التي صاحبت انتشار الفيروس لتجناح هي الأخرى حواجز الزمان والمكان، اجتياح مكاني جعل من غياب الحواجز المكانية الثابتة مثارا للارتقاء إلى عوالم مختلفة عن طريق شبكات الانترنت الفسيحة واجتياح زماني امتلك أدوات التخلص من روتين الذهاب والإياب ومزاحمة الآخرين بحثنا عن سرعة الوصول إلى حيز مكاني ربما كان أضيق مما تتحملة رحابة العقول.

وبكل ما يمتلكة التعليم عن بعد من موارد سمعية وبصرية ورسوم توضيحية وصور متحركة تحول التعليم عن بعد من أسلوب التلقين إلى أسلوب تفاعلي مصحوب بمؤثرات بصرية وسمعية تجعل من العطلية التعليمية الجامدة عملية أكثر جذبا، وتساعد الطلاب إلى الدخول إلى المحتوى دون التوقف عند عنبات رائحة الأوراق، وهو ما سارعت وزارة التربية والتعليم الفني في مصر بالتوجه إلبه من خلال بنك المعرفة المصري كوسيلة للتغلب على تعليق الدراسة وقد قدمت منظمة اليونسكو بعض البرامج التي تساعد على تصميم المقررات و المهمات والو اجبات و الاختبار ات وتصحيحها إليكترونيا و التو اصل مع الطلاب من خلال بيئة افتر اضية من خلال تطبيقات يتم تحميلها عن طريق الهواتف الذكية مثل برنامج "بلاك بورد، برنامج ادمودو "وتطبيق جوجل كلاس روم، يساعد ويسهل التواصل بين المتعلمين ومعلميهم وقد استخدمنة جامعة القاهرة، مما سبق نرى أن هنالك ضرورة ملحة من استخدام التعليم عن بعد أو التعليم الإلكتروني عن طريق الانترنت، وهذا أصبح شيئا واقعيا، وبالتالي سوف يؤثر ذلك على أداء الأنشطة التربوية المختلفة سواء الصفية أو اللاصفية في المدرسة، وكان حتما الوصول إلى طريقة أخرى من أجل حصول تلاميذ مرحلة التعليم الأساسي على هذه الأنشطة في ظل أزمة كورونا وفي ظل الأعداد الكبيرة من التلاميذ وانخفاض المساحة المطلوبة لأداء هذه الأنشطة في ظل التباعد الاجتماعي. وحيث إن هنالك مبررات كثثرة لعملية تحويل الأنشطة الصفية واللاصفية إلى إلكترونية وذكية منها توفير الوقت والجهد على الطالب وولي الأمر من خلال عدم اللجوء للمكتبات والمتخصصين بأعداد الأنشطة والمشاريع المدرسية، كما أنها تتيح الاتصال الفعال بين الطلبة أنفسهر وبينهم وبين المعلم، ومن أهم مميز اتها أيضا شعور الطلبة بالمساواة، حيث إن أدوات الاتصال الإلكتروني والذكي تتيح لكل طالب فرصة التعبير عما يجول بخاطره من انفعالات وأحاسيس دون التعرض للإحراج كما تتيح وسائل الاتصال الإلكتروني والذكي سهولة وصول الطالب إلى المعلم في أسرع وقت حتى خارج أوقات الدراسة، وذللك من خلال إرسال النشاط المطلوب منه ويمكن المعلم من تقديم التغذية الر اجعة للطالب في أي وقت أيضا، كما تلعب دورا في تقليل الأعباء الإدرارية بالنسبة للمعلمين من خلال إمكانية الإرسال والاستلام عن طريق الأدوات الإكترونية مع إمكانية معرفة استلام الطالب لجميع الأنشطة والواجبات المطلوبة منه، وإن الأدوات الإكترونية تلعب دورا هاما في تقليل حجم العمل في المدرسة من خلال توفير أدوات تقوم بتحليل نتائج الأنشطة و الو اجبات المختلفة وحتى الاختبار ات وأعداد إحصائيات بذلك، وسهولة إرسالها لمن يهمه الأمر من أولياء الأمور أو إدارة المدرسة.[ 1] 
و إن هناك ضرورة ملحة إلى اعتماد المدارس الكلي على الوسائط الالكترونية الذكية ولعل من أنجح الاستر اتيجيات في هذا المجال هو استراتيجية الصف المقلوب التي جعلت التعلم أكثر متعة وفاعلية للطالب وأعادت للمعلم دورة الأصيل في تعديل سلوكيات الطلاب من خلال عملية التعلم، كما أن تدريب الطلاب عن طريق البحث الصحيح عن المعلومات وآلية التوثيق الجيد لهذه المعلومات، كما يجب تعزيز التو اصل بين الأسرة والمجتمع المدرسي لتوحيد آلية تنفيذ هذه الأنشطة وضرورة أن يكون ولي الأمر على دراية بالاستر اتيجيات التي تنتهجها المدرسة في هذا المجال وكذلك تخصيص أوقات محددة للتفيذ كما يجب على المدرسة تفعيل وسائل التو اصل الأخرى و عدم الاعتماد الكلي على التقنيات، وذلك من خلال الرحلات العلمية والترفيهية وتبادل الزيارات بين المجتمعات التعليمية والخروج بالبيئة التعليمية خارج إطار الصف الدراسي، حيث إنه لاضرر من تحويل غالبية الأنشطة اللاصفية إلى إلكترونية وذكية ما دام ذلك وفق أطر صحيحة بالتز امن مع تنفيذ أنشطة أخرى تعزز من طرق التواصل المختلفة من قبل الأسرة المدرسية.[2] وحيث تعد الأنشطة التعليمية من الملامح العامة والمميزة لمواد التعلم عن بعد والتي تساعد على التعلم النشط وتشجيع الطلاب على البحث و التفاعل أثناء عملية التعلم فمها كان طبيعة المحتوى، فإنها ستكون أكثر فعالية إذا جعلنا الطالب ودفعناه ليكون متعلما نشطاً إيجابياً لا مجرد مستقبل، ومن حيث تلعب الأنشطة دورا جو هريا في تحديد نواتج التعلم فهي تحدد كيف سيقوم الطالب بالإندماج مع المحتوى [3] التعليمي وبناء المعرفة. وحيث إنه لاتوجد نظرية تعلم واحدة يمكن الاعتماد عليها حصريا في تصميم الخبرات التعليمية وتحقيق أهداف التعلم المختلفة فالنظريات السلوكية تتعامل مع السلوك الظاهري للمتعلم والذي يخضع للملاحظة والقياس دون النظر إلى العمليات العقلية ور اء حدوث هذا السلوك، بينما يهنم أصحاب النظرية المعرفية بالعمليات العقلية التى تحدث داخل عقل المتعلم وينتج عنها سلوكة وتقوم النظرية البنائية على أن المعرفة تبنى بو اسطة المتعلم وتشجع النظرية الاتصالية بناء الخبرات والتفاعل الاجتماعي عبر الثبكات ويمكن الاستفادة من جوانب القوة في كل نظرية للوصول إلى جودة تصميم الأنشطة الإكترونية.[4] وتتضح مشكلة البحث في أن هناك حاجة ماسة لتصميم مجمو عة من الأنشطة التعلم الإلكثرونية وذلك لدمجها مع طريقة التعلم التقلبدية، وذللك في ظل التعليم عن بعد، وتتضح مشكلة البحث في الإجابة على الأسئلة التالية:

$$
1 \text { - ما هو واقع تطبيق الأنشطة التربوية الصفية الإكترونية في مدارس التعليم الأساسي؟ ها هي التحديات التي تواجهة تطبيق الأنشطة الصفية الإلكترونية في مدارس التعليم الأساسي؟ }
$$


ـالتعرف على واقع تطبيق الأنشطة الصفية الإلكترونية في مدارس التعليم الأساسي. ـالتعرف على التحديات التي تواجهه نطبيق الأنشطة الصفية الإلكترونية في مدارس مرحلة التعليم الأساسي. أهمية البحث: تعد الأنشطة الصفية الإلكترونية الرافد المهم للرسالة التربوية، وتستهف تزويد التلاميذ بالجوانب المعرفية والتعليمية لتحقيق نمو الشخصية و إعداده لحياة أفضل وتوجيهه نحو السلوك الإيجابي، وإن الأنشطة داخل المدرسة أو خارجها من أنشطة تعلم وتعليم طالما تتم تحت المدرسة لتحقيق أهدافها أو أهداف المجتمع من خلالها، وهي تطبيق لمفهوم النشاط الذي يعني أن النشاطات سواء أكانت بدنية أو عقلية ضرورية للتعلم و أيضا تساعد الأنشطة التربوية الإلكترونية المدرسة فى بث الإيجابية والحماس في شخصية المتعلم وبروز مشاركتة الفعلية في اقتر اح وتخطيط وتنفيذ وتقويم ما يحتاجة من خبرات وكذلك يساعد البحث في حث المعلمين في مدارس التعلم الأساسي في تصميم الأنشطة الصفية الإكترونية. حدود البحث: ـ حدود بشرية: تحدد البحث بـ ( 120) معلم ومعلمة من مرحلة التعليم الأساسي، (50) ولي أمر ، (15) مدير مدرسة، (30 ) مشرف نشاط. حدود مكانية: اشتمل هذا البحث على 10 مدرسة إعدادي، و5 مدارس ابتدائي بإدارة أبو كبير التعليمية بمحافظة الشرقية. حدود زمنية: تم إجر اء هذه الدراسة في العام الدراسي 2021/2020 م الفصل الدر اسي الأول. مصطلحات البحث:

\section{الأنشطة الصفية الإكترونية:}

تعرف بأنها جميع الأعمال التي يمكن أن يقوم بها المتعلم من خلال تفاعله أو تعلمه النشط على شبكة الانترنت، ويمكن أن تكون هذه الأنشطة غير منز امنة ولها أهداف محددة ويمكن أن تتم من خلال رسائل إلكترونية يرسلها المعلم إلى طلابه.][5 تعرف إجرائيا: هي عبارة عن مجموعة من الأنشطة المصممة باستخدام تطبيقات مستحدثات التكنولوجيا مثل: الانترنت، البريد الإكترونى، غرف المحادثة، وسائل الاتصال الاجتماعي، من قبل معلمي مدارس التعليم الأساسي. 


\section{مفهوم الإنشطة الإكترونية:}

ترى سالمون Salmon,2002 أن الأنشطة الإلكترونية تعطي إطار التحسن التفاعل والمشاركة في التعلم الإلكتروني وتعمل على خفض تكاليف التعلم، ولابد أن تكون الانترنت ضمن خطة تتفيذها وتستخدم فيها لوحات المناقشة لتشجيع عملية الاتصال والتفاعل بين المتعلمين، كما يمكن زيادة فاعلية الأنشطة إذا تمت مراعاة مبادىء تصميم التعلم البنائي، ومنها ضرورة تفاعل المتعلمين من خلال الأنطة التي يقومون بها أثناء عملية التعلم، فالمتعلم يمارس الأنشطة في معالجته للمعلومات، وهذا يساعد على تغيير أو تعديل البنية المعرفية للمتعلم، ويعمل على تحسينها وتطوير ها. فعندما يبذل المتعلم جهاً عقليا هذا يساعده على اكتشاف المعرفة بنفسة خاصة عندما palma \& pitera ) يو اجهه مشكلة تعليمية ويقوم باقتر اح لحلها وفرض العديد من الفروض و البدائل لحلها. وكذلك يشير بالما وبيترا 2008, ) إلى أنه لا بد الأخذ بعين الاعتبار نظريات ونماذج التعلم الإلكتروني عند التفكير في تصميم المحتوى الإلكتروني، وأن يكون التعلم مرنا لتتيح للمعلم السبيل لتوظيف العديد من الأنشطة الإلكترونية المتزامنة واللامتزامنة حتى يمكن متابعة طلابه، والرد على

كما أوضح كرينور وبلوتر (Creanor\&Bulter,2004) أن الأنشطة الإلكترونية هي الطرق والأساليب المختلفة التي تعتمد على النشاط الذاتي والمشاركة الإيجابية بين الطلاب فمن خلالها يقوم الطالب ببعض العمليات المعرفية كالملاحظة والبحث في التوصل إلى المعلومات المطلوبة بنفساه، وبتوجيه من المعلم وتحثه على الإبداع و الاستنتاج.]7]

\section{أنواع الأنشطة الصفية الإكترونية:} يوجد العديد من الأنشطة الإكترونية التي يمكن تنفيذها من خلال البيئة الافتر اضية للتعلم مثل: - أنشطة تنمية المهار ات القر اعة و الكتابة من خلال أنشطة المشاهدة و الاستماع. - أنشطة تتمية المهار ات الاجتماعية من خلال توظيف أدوات التو اصل الاجتماعي. ـ - أنشطة لتنمية المهار ات التفكير العليا من خلال أنشطة المناقثات و الأسئلة المفتوحة وتبادل الآراء و الخبراء. - عمل العروض الإلكترونية عن عناصر المحتوى وعرضها إلكترونيا للزملاء واستلام التغذية الراجعة. - التعلم من خلال الإنترنت وتبادل المعرفة عبر وسائل الاتصال و التفاعل الموجودة في بيئة التعلم الإلكتروني.[8]

\section{ما هي المتطلبات التربوية لتطبيق الأنشطة الإكترونية: ـأن تحقق الأنشطة التعليمة الأهداف المرجوة منها. -أن يكون المحتوى مدعما بأنشطة متنو عة.}


-أن تكون الأنشطة التعليمة منظمة بطريقة منطقية. ـأن تتفق الأنشطة التعليمية المقدمة من خلال التعلم الإلكتروني مع الأنشطة والممارسات التدريسية المقدمة في داخل الفصول الدراسية. ـأن تعرض الأنشطة بطريقة تثير تفكير المتعلمين، وتساعدهم على التفكير الناقد والابتكاري. ـأن تعرض الأنشطة بطريقة تشجع على التعلم التعاوني، وتسمح للمتعلمين رفع بناء المعلومات. ومن الأنثطة التبي يجب على المعلمين بالمدارس أن يتمكنوا من تصميمها وتطبيقها ومن تلك الأنشطة الإكترونية ما يلي: تشغيل نظام Moodle لإدارة المقررات الإلكترونية.

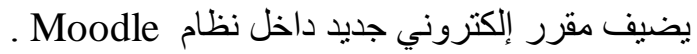
ينشئ محتوى صفحة نصية إلى واجهة المقرر الإلكتروني. ينشئ محتوى صفحة ويب إلى واجهة المقرر الإلكتروني.

ينشئ رابطا لموقع ويب إلى واجهة المقرر الإلكتروني. ينشئ مجلدا لعرض ملفات المتعلمين بالمقرر الإلكتروني. ينشئ حزمة إصدار IMS إلى واجهة المقرر الإلكتروني. يدرج ملصقة إلى واجهة المقرر الإلكتروني. يستخدم الكتل Blocks المتاحة بالمقرر الإلكتروني. يدرج مهمة Assignment من نوع تحميل ملف واحد. يدرج مهمة Assignment من نوع النص المباثر. Assignment من نوع دئر يدرج مهمة Assignment من نوع تحميل نشاط بدون اتصال. ينشئ غرفة حوار Chat إلى و اجهة المقرر الإلكتروني.

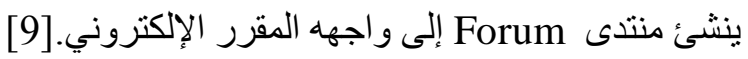

تصميم الانشطة التعليمية الإكترونية: يمكن القول إن عملية تصميم الأنشطة التعليمية الإلكترونية تستند إلى النظرية البنائية والمعرفية والاتصالية وتتضح، أبعادها في الآتي: 1-البعد المعرفي: ويعبر هذا البعد عن البنية المعرفية للمتعلم وتوافر قدرتة على تحويل وتغيير البنية المعرفية الحالية وتنظيم المعلومات الجديدة مع المعلومات السابقة، أي النمو الذهني من خلال قدرة المتعلم على استنباط وتكوين رؤية معرفية لمجالات و أبعاد محتوى التعلم من شأنها إحداث التر ابط و التكامل والتمايز بين أبعاد المحتوى، وهذا الوعي المعرفي يعد نقطة الانطلاق لفهم المحتوى التعلم وبناء المعنى، و هذا ما دعت إليه النظرية البنائية التي تسعى إلى دراسة أساليب بناء المتعلم رؤيتة الثخصية للعالم من حوله بالاستناد إلى خبر اتة السابقة و أنشطته المتعددة. 2-البعد الاجتماعي: من بين الاعتبار ات الضرورية لضمان جودة الأنشطة التعليمية في بيئات التعلم الإلكتروني، ويقصد به تدعيم البناء الجماعي للمعرفة من خلال التفاوض الاجتماعي، وليس من خلال التنافس بين الطلاب بعضهم البعض، ويتمثل في بناء مجتمعات التعلم 
الاتصالية التي تسعى لوضع التعلم عبر الثبكات في إطار اجتماعي فعال، والتركيز على نشاطات التعلم التفاعلية لتشجيع مستويات التفكير العليا مع توفير التفاعل الاجتماعي للطلاب و المعلم بصور مختلفة.

3-البعد العقلي: يمثل البعد العقلي أهمية بالغة في بناء المعرفة من خلال بناء نماذج وتصورات عقلية تساعد في استخدام أنثطة التعلم وتعميمها على مو اقف متتـابهة بهدف انتقال وتوسيع أثر التعلم، وهنا تظهر أطر النظرية المعرفية فتهنم بدراسة العمليات العقلية التي ينتج عنها السلوك لذا تقدم أنشطة تعليمية إلكترونية تسمح بالتجريب والاكتشاف و التنويع و التعديل في سلوك المتعلم .

4-البعد الثخصي الذاتي: من بين الأبعاد التي ينبخي أخذها في الاعتبار البعد الثخصي، ويعبر هذا البعد عن الجانب النفسي للمتعلم و التفاعل مع الذات وتطور ها وهذا ما تتادي به النظرية البنائية، ويقصد به الوعي الذاتي والتطور الشخصي، لذا ينبخي مر اعاة جانب الحضور النفسي والتفاعل الذاتي للمتعلم واكتساب قدرات ومهار ات والذي يتميز بأنه نشط وبنائي ومقصود وأصيل وتعاوني، كما أن البيئات التعليمية الإلكترونية و الافتراضية صالحة للتعلم البناء شخصية منها: المثابرة_الثقة فى النفس- التغلب على الصعوباتـ- إدارة الوقت_المبادرة-الاعتماد على النفس-الاستقلاليةـ التعبير عن الرأي-إدارة الذاتـ دافعية الذات، ونخلص من ذلك إلى أن عملية بناء المعرفة في عقل المتعلم يتضمن تحكمه وتأمله فيما يتعلمه، وأن بناء المعرفة عملية تفاوضية اجتماعية، وهو التعلم الهادف.[10] أنواع الأنشطة الإكترونية في التعلم الإكتروني. تُعد الأنشطة الإلكترونية أحد أساليب التعلم النشط التي يمكن استخدامها وتوظيفها من خلال بيئة التعلم عبر الويب ومنها:التعلم التعاوني فى مجمو عات عبر الويب، المناقشة الإكترونية، العصف الذهني الإلكتروني، المنتديات الإلكترونية، لوحات النقاش الإلكترونية، التو اصل عبر الميل، كما تتعدد وتتنوع الأنشطة التي يتضمنها كل أسلوب فمثلاً المناقثنة الإلكترونية قد تتضمن أنشطة منز امنة مثل المحادثة واستخدام غرف الدردشة، أو المؤتمرات عن بعد، وقد تتضمن أنشطة غير متز امنة مثل استخدام البريد الإلكتروني أو لوحات النقاش، كما أن أسلوب العصف الذهني ينطلب استخدام العديد من الأنشطة الإكترونية عبر الويب مثل استخدام مجموعات البريد الإكتروني أو المنتديات أو استخدام بر امج التفاعل عبر الويب والمدونات.[11 و أثشار علي بن شرف الموسوي[12] إلى أن هناك تجدد مستمر لأشكال استخدام تقنيات التعليم والمعلومات في تصميم وتنفيذ الأنشطة التعليمية، ويمكن استقصاء أكثر ها أهمية فيما يلي: - الرسوم والملصقات التوضيحية والكرتونية: وهي من الوسائط التي تحض الطلاب على التفكير الناقد من خلال قراءة هذه الملصقات الرسوم وتحليلها ومقارنتها. 
ـ ـ البرمجيات التعليمية المتوفرة على الإتترنت: من خلالها يتم تقديم مجمو عة من الأنشطة والتدريب و الممارسة التي تقدم للطلاب باستخدام الحاسوب بقصد إحداث تغير ات في السلوك يؤدي إلى تشكل مهار ات التفكير والاستتناج في مو اقف تعليمية الكترونية أو مدمجة

$$
\text { في التعليم التقليدي الصفي. }
$$

ـ ـ المكتبات الرقمية والإكترونية: ويمكن من خلال هذه المكتبة تحسين الدعم المقدم للأنشطة الصفية و اللاصفية داخل وخارج غرفة

ـ ـ البريد والمنتديات الإكترونية: حيث يتم استخدامهما لدعم الأنشطة التعليمية الذاتية والتعاونية في مجالات البحث وطرقه ومر اجعة المقالات البحثية للطلاب إضافة لاستخدامهما في تحديد مو اعيد تسليم الأنشطة المكتملة والتعيينات الصفية. ـ المختبرات الافتراضية: وهي عبارة عن مختبرات مصممة على برمجيات ثلاثية الأبعاد ويقوم الطالب باختبار نوع التجربة العلمية و أدو اتها وطريقة إجر اءها ويتفاعل معها خلال عمليات التجريب.

ـ الألعاب التعليمية العادية والمحوسبة والإكترونية: حيث تقوم بدور مهم في تعليم الصغار من خلال الأنشطة التعليمية المصممة بصورة جيدة وممتعة عليها، ومن أمثلتها: البطاقات المصورة، وألعاب التركيب، و الألعاب الإلكترونية المتطورة، حيث يمكن دمجها كجز ع من الأنشطة التعليمية أو بناء الأنشطة التعليمية على أساسها. المواقع والمصادر الإكترونية : حيث يمكن دمجها كجزء من الأنشطة التعليمية أو بناء الأنشطة التعليمية على أساسها، وقد تتعدد وتتنوع الأنشطة التي يتضمنها كل أسلوب فمثلاً المناقشة الإلكترونية قد تتضمن أنشطة متز امنة مثل المحادثة واستخدام غرف الدردشة، أو المؤتمر ات عن بعد، وقد تتضمن أنشطة غير منز امنة مثل استخدام البريد الإلكتروني أو لوحات النقاش، كما أن أسلوب العصف الذهني يتطلب استخدام العديد من الأنشطة الإلكترونية عبر الويب مثل استخدام مجموعات البريد الإكتروني أو المنتديات أو استخدام برامج التفاعل عبر الويب والمدونات.

بناء الأنشطة التعليمية التقتية: تتضمن خطوات بناء الأنشطة التعليمية باستخدام التقنيات المر احل الرئيسية التالية: مرحلة التحليل وفيها يقوم المعلم أو المصمم بعمل الخطوات التالية: - إعداد رؤية للنشاط التعليمي من حيث أهدافه العامة و التفصيلية. ـ تحليل احتياجات الطلاب وخصائصهم من حيث تحديد أعمار هم ومستوياتهم الفكرية وقدر اتهم المهارية وخلفياتهم الثقافية و إمكاناتهم العملية وميولهم ورغباتهم. 


\section{ـ تحليل الأهداف السلوكية للنشاط ويقوم بتجزئتها وترجمتها إلى أهداف صغيرة.}

ـ تخطيط محتوى النشاط وتحديد المهام المطلوبة فيه وتجزئتها بشكل يمكن تحويلها إلى صيغة تقنية.

ـ تحليل عمليات الانتاج المتوقعة فيحدد المتطلبات التقنية واختيار نوعية التقنية منل: البرمجية المحوسبة أو الموقع الإلكتروني واختيار وسائط الاتصال التعليمية لهذه التقنية والتي تستخدم أو تتنج لايضاح المفاهيم والأفكار وشرح محتوى الأنشطة التعليمية. ـ [13] - تحديد التكلفة الإجمالية لعمليات الإنتاج.

\section{ـ مرحلة التصميم وفيها يقوم المعلم أو المصمم بعمل الآتي:}

ـ تصميم محتوى الأنشطة التعليمية.

- إخر اج المحتوى العلمي للأنشطة بحيث يوضع بتتابع سلس للأفكار و المفاهيم والنظريات والقوانين بأسلوب ير اعي قدرات الطلاب. تصميم المحتوى التدريبي فنيا وجماليا على صورة مواد تعلم وسائطية تمكن الطلاب من التعلم الذاتي والموجهة.

ـ تطبيق مبادىء تصميم الوسائط الفنية والنفسية والتربوية في تصميم التقنية المختارة بحيث يتم جذب الانتباه وتعزيز الذاكرة وتكرار المعلومات واستثارة النشاط الإيجابي للمتدرب.

\section{ـ مرحلة التطوير وفيها يقوم المصمم أو المعلم بعمل الآتي:}

- وضع السناريو هات و المخططات الإنسانية للخطوات التطبيقية للإنتاج و التنفيذ الفعال لمختلف منطلبات الأنشطة التعليمية. - وضع الأجندة التفصيلية مقرونة بالجدول الزمني للإنتاج التقنية المختارة. ـ تحويل الخطة إلى شكل تخطيطي مصور يوضح الإجراءات اللازمة حتى إكمال العمل. مرحلة الإنتاج وفيها يقوم المعلم أو المصمم بعمل الأتي:

- إنتاج مو اد التعلم الوسائطية حيث تدمج في محتو اها كافة المكونات الوسائطية التفاعلية.

ـ تحويل المحتوى النصي إلى قو الب متعددة ووسائط متحركة وتقوم على التفاعلية و الأدائية وتوضح الأشكال و الرسوم المثيرة لدافعية المتدرب وجاذبية الانتباه، وتكون كل مادة تعليمية وسائطية مونا لنشاط تعليمي كامل وقائم بذاتة كما ينبغي عند إنتاج هذه المواد مر اعاة معايير الجودة الضرورية لضمان النوعية القياسية للمنتج.[14] 
مرحلة التقويم وفيها يقوم المعلم أو المصم بعمل الآتي: ـ قياس آداء المدربين والمتذربين ومدى تأثير هذه التقنية في تحسين معارف ومهار ات وكفايات الطلاب. ـ استخدام التقويم البنائي و الختامي لقياس مستوى الإنجاز والآداء التعليمي ومدى تحقيق الأهداف التعليمية. ـ استخدام استطلاعات الرأى او الاستييانات لتعرف اتجاهات ومو اقف المتدربين نحو الأنشطة التعليمية التقنية.

نخلص من ذلك: أن الأنثطة التطليمية مجال مهم لتطوير شخصية الطالب و إثراء خبراته واكتساب المهارات و اتقانه إياها، ولا بد إذاً على المعلم أن يضمنها في تدريسه، ومع التطور الحادث في تقنيات التعليم و المعلومات ووسائطها سيصبح استخدامها في الأنشطة بل إنها تضفي جوا من التشويق على طرق التنديس والتعلم، حيث إنها إذا ما حسن استخدامها لتقلل الوقت والمال المبذول في تصميم العلية التعليمية.[15

\section{بحوث سابقة}

هدف بحث ويفر (weaver,1977)] [16] إلى النعرف على مكانة الأنثطة المدرسية اللاصفية ومكانتها فى مناهج الدرسة و على مدى الاهتمام بها، وقد اقتصرت على الأنثطة الصفية فقط في مر احل التعليم المختلفة لذا جاءت هذه الدراسة لتبين واقع هذه الأنشطة وصعوبته تنفيذها وأثرها على التحصيل وتم التوصل إلى أن هناك بعض المعوقات المرتبطة بالامكانات المادية والبشرية و وعد أخذ الأنشطة في الحسبان عند تقويم التلاميذ. هدف بحث (زهو ,2008) [17] إلى التعرف على واقع ممارسة الانثطة المدرسية وتحليل دور هذه الانثطة في تتمية الإبداع من خلال آداء المعلم لممارسة النشاط الددرسي، ووضع تصور مقترح لتفعيل دور الأنثطة المدرسية في تتمية الإبداع واستخدت الدراسة المنهج الوصفي، واستخذت الباحثة بطاقة ملاحظة وتوصلت الدراسة إلى أن المعلمات في الدارس الخاصة حديثي التخرج أكثر حرصا على الأنشطة الإبداعية من معلمات الدارس الحكومية، وارتفاع نسبة الاهتمام بالأنشطة في المدارس الخاصة عن المدارس الحكومية ،وإن تكس المناهج يعوق القيام بالأنشطة الطلابية .

هدف بحث (هاني ابر اهيم ومحمد علي ,2007) [18] إلى التعرف على دور الأنثطة الطلابية في تتمية بعض السمات الإيجابية لاى طلاب جامعة جنوب الو ادي، واستخدم الباحث المنهج الوصفي وتكونت عينة البحث من 400 طالب وطالبة ، ونوصل إلى النتائج التالية: إن الطلاب أكثر تميزا في السمات الذاتية والثقافية والسياسية فى حين أن الطالبات تميزن في السمات الأخلاقية، و لاتوجد أي فروق بينهم في السمات الاجتماعية، كما توصلت النتائج إلى أن المشاركين في الأنثطة أكثر تميزا عن المشاركين. 
هدف بحث هويدا عبد الحميد (2020) [19] التعرف على أثر اختلاف نمط ممارسة الأنشطة الإكترونية فردي/جماعي ضمن بيئة التعلم المعكوس في تتمية الأداء التقني والثقة بالنفس لاى طلاب تكنولوحيا التعليم، وتكونت عينة البحث من 60 طالبا وطالبة بالفرقة الر ابعة تكنولوجيا تعليم ـكلية التربية النوعية ــامعة عين شمس، تمّ تقسيمه إلى مجموعنين وتم التوصل إلى أن ممارسة الأنشطة الفردية الإلكترونية لها أثر في الأداء التقني و الثقة بالنفس لدى الطلبة.

بحث أحمد النوبي ونادية التازي (2019) [20] التعرف على مواصفات الأنشطة الإكترونية التي يمكن أن تقدم للتلاميذ ذوي صعوبات التعلم، فاعلية أنشطة التعلم الإلكتروني في تحسين التحصيل الدراسي، وتكوينت عينة البحث من 8 تلاميذ ذكور و4 تلاميذ أناث من ذوي صعوبات التعلم، بعد تطبيق الاختبارات التشخيصية اللازمة والتي تكثف عن صعوبات تعلم القر اءة واستخدم المنهج التجريبي بتصميم شبه تجريبي، واستخدم اختبار مهار ات القراءة، وتوصل إلى النتائج التالية وجود أثر إيجابي لاستخدام أنتطة التعلم المدمج على تحسين التحصيل ومهارة القراعة لدى التلاميذ ذوي صعوبات التعلم. إجراعات البحث: منهج البحث: استخدمت الباحثة المنهج الوصفي التحليلي في جمع البيانات والمعلومات عن طريق استبانه تم تصميمها لهذا الغرض ومن ثم تحليل البيانات والمعطيات المناسبة واستخلاص النتائج و التعليق عليها.

\section{عينة البحث:}

- - تكونت عينة البحث من (120) معلم ومعلمة من مرحلة التعليم الأساسي، (50) ولي أمر، (15) مديرمدرسة، (30 ) مشرف نشاط.

أدوات البحث وخصائصها السيكومترية: هي استبانه مقسمة إلى اثنان من المحاور: المحور الأول منعلق بواقع تطبيق الأنشطة الصفية الإلكترونية، والمحور الثاني متعلق بالتحديات التي تو اجه تطبيق الأنشطة الصفية الإكترونية في مدارس التعليم الأساسي، ومن أجل التأكد من صدق أداة البحث تم عرضها على مجمو عة من المحكمين في كلية التربية، وقد أبدى السادة المحكمين وجهة نظر هم وملاحظتهم وتم الأخذ بها. وقد قامت الباحثة بحساب الصدق والثبات: ـ الصدق: تم حساب الصدق بالطرق الآتية:

ـ صدق المحكمين: وتم التحقق من صدق الاستبيان بعرضة على مجموعة من المحكمين والمتخصصين في القياس والتقويم و عددهم عشرة محكمين، وكانت نسبة الاتفاق عليها 95\% على أن الاستبانه صالحة لاستخدامها في هذا البحث. 
صدق الاتساق الداخلي: نم حساب الاتساق الداخلي للعبار ات وذلك بايجاد معاملات الارتباط بين الدرجات التي حصل عليها

$$
\text { المفحوص في كل عبارة من عبار ات الاستبانه والدرجة على البعد. }
$$

ثبات الاستبيان:

تم حساب ثبات الأبعاد الفرعية لاستبيان الأنشطة التربوية "' : بطريقتين: الأولى عن طريق معامل ألفا لـ كرونباخ، و الثانية عن طريق

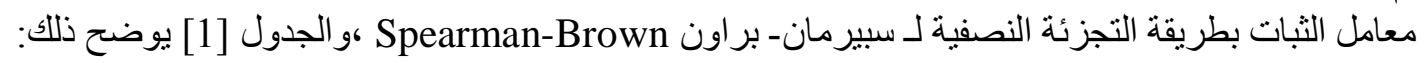
جدول(1):عاملات ثبات الأبعاد الفرعية والثبات الكلي لاستبيان "الانشطة الصفية الاكترونية"

\begin{tabular}{|c|c|c|c|}
\hline معامل ثبات التجزئة النصفية & معامل ثبات كرونباخ & عدد المفردات & الأبعاد \\
\hline .52 & .56 & 15 & البعد الأول: و اقع تطبيق الانشطة التربوية فى \\
\hline .57 & .65 & 15 & تطبيق الانشطة الثناني: التحديات و الفرص التي التي تواجها \\
\hline
\end{tabular}

- يتضح من الجدول (1) أن معاملات الثبات تراوحت بين(52. إلى 67.) و هي معاملات ثبات مرتفعة ودالة احصائيًا مما يسمح باستخدام هذا الاستبيان في الدر اسة الحالية.

الاتساق الداخلي بين الابعاد والدرجة الكلية للمقياس:

تم حساب الاتساق الداخلي بين الأبعاد والدرجة الكلية الاستبيان:عن طريق معاملات الارتباط بين درجة كل بعد من استبيان الأنشطة التريوية و الدرجة الكلية الاستبيان و الجدول التالي يوضح ذلك:

جدول(2): معاملات الارتباط بين درجة كل بعد من استبيان " الأنثطة الصفية الاكترونية " والدرجة الكلية للاستبيان

\begin{tabular}{|c|c|c|c|}
\hline مستوي الدلاكة & الارتباط & الأبعاد & s \\
\hline 0.01 & $* * .67$ & الأبعد الأول: واقع تطبيق الأنشطة الصفية الإكترونية في المدارس & 1 \\
\hline $\mathbf{0 . 0 1}$ & $* * .65$ & الألكترونيةً في الثمدي: التحدات التي تواجهانة تطبيق الانشطة الصفية & 2 \\
\hline $\mathbf{0 . 0 1}$ & $* * .68$ & الارجة الكلية & 3 \\
\hline
\end{tabular}

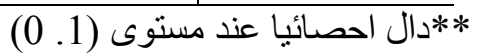

ويتضح من جدول (2) أن قيم معاملات الارتباط مرتفعة ودالة عند مستوى (0.01) مما يدل على وجود اتساق داخلي مرتفع

لأبعاد الاستبيان، ومن ثم فإن أبعاد الاستبيان تتمتع بدرجة عالية من الاتساق الداخلي، مما يدل على صدق وثبات المقياس

وصلاحيتة في قياس مستوى تطبيق الأنشطة الإكترونية. 


\section{نتائج البحث ومناقشتها:}

للاجابة على السؤال الأول الذي ينص على: ما واقع تطبيق الأنشطة الصفية الإلكترونية في مدارس التعليم الأساسي، تم استخدام اختبار "ت" لعينة واحدة، ويوضح الجدول التالي نتائج اختبار "ت" للبعد الأول من استبيان تطبيق الأنشطة الإلكترونية.

جدول(3): نتائج اختبار "ت" لفقرات البعد الأول من استبيان تطبيق الأنشطة الصفية الإلكترونية

\begin{tabular}{|c|c|c|c|c|}
\hline مستوى & 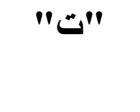 & الانحراف & المتوسط & 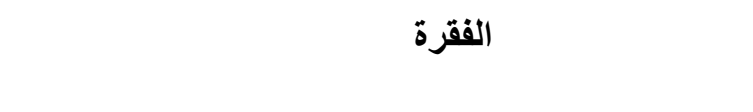 \\
\hline 0.01 & 11.68 & .594 & 1.49 & الخاصة بالأنشطة الإلكترونية المادية ونقص التجهيزات والمو اد \\
\hline 0.01 & 11.63 & 0626 & 1.52 & ملائمة الفصول لتنفيذ للنشاط الإلكتروني \\
\hline 0.01 & 12.95 & .622 & 1.57 & يوجد حوافز للمعلمين القائمين على الأنشطة الإلكترونية \\
\hline 0.01 & 13.051 & .607 & 1.56 & الصفية الإلكترونية الكبرة لدى المعلمين فى تنفيذ الأنشطة \\
\hline 0.01 & 9.584 & .679 & 1.46 & يتوفر الوقت الكافي لممارسة الأنشطة الإلكترونية \\
\hline $\mathbf{0 . 0 1}$ & 10.260 & .641 & 1.47 & ترتبط الأنشطة الإلكترونية بالمنهج المدرسي \\
\hline 0.01 & 12.306 & .632 & 1.55 & المجموع تخصيص درجات للنشاط الإلكتروني و لا تضاف إلى \\
\hline 0.01 & 9.191 & .669 & 1.44 & يوجد اهتمام مديرين المدارس بأهمية النشاط الالكترونى \\
\hline 0.01 & 10.884 & .650 & 1.50 & يوجد قلة الو عي بأهداف النشاط الإلكتروني \\
\hline $\mathbf{0 . 0 1}$ & 11.686 & .593 & 1.49 & الطلاب تمتنع من المشام الوقت في اليوم الدراسي بالمقرر في برنات يجعل النشاط الإكتروني \\
\hline $\mathbf{0 . 0 1}$ & 10.260 & .641 & 1.47 & يهتم السادة أولياء الأمور بالأنشطة الإلكترونية المختلفة \\
\hline $\mathbf{0 . 0 1}$ & 10.667 & .613 & 2.58 & الدراسة السادة أولياء الأمور بأن النشاط الإلكتروني يعطل \\
\hline $\mathbf{0 . 0 1}$ & 14.324 & .650 & 2.49 & فقط و لايهتمر اهتمام التلاميذ المتفوقين على الدروس اليومية \\
\hline $\mathbf{0 . 0 1}$ & 13.257 & .649 & 2.48 & تحصنتع التلاميذ بأن النشاط الإلكتروني يؤثر سلبا على \\
\hline
\end{tabular}

يتضح من الجدول السابق: أن جميع قيم "ت" دالة احصائيا كما أن جيع قيم المتوسطات والأوزان النسبية لجميع الفقرات لهذا البعد أقل من (2) بدرجة متوسطة وقريبة من (1) أى أن هذه العبارات على درجة قليلة من الموافقة على مدى تطبيق الأنشطة الصفية الإلكترونية 
في مدارس التعليم الأساسي، واتضح ذلك في بحث (زهو ,2008) حيث هدف إلى التعرف على واقع ممارسة الأنشطة المدرسية وتحليل دور هذه الأنشطة في تنمية الإبداع من خلال أداء المعلم لممارسة النشاط المدرسي، وقد توصل البحث إلى أن المعلمات في المدارس الخاصة حديثي التخرج أكثر حرصا على الأنشطة الابداعية من معلمات المدارس الحكومية وارتفاع نسبة الاهتمام بالأشطة في المدارس الخاصة عن المدارس الحكومية، وإن تكدس المناهج يعوق القيام بالأنشطة الطلابية، وتتقق هذه النتيجة مع ما توصلت إليه الباحثة، وترى الباحثة أن ذلك يرجع أيضا إلى اعتقاد أولياء الأمور أن الأنشطة الإكترونية تؤثر سلبيا على تحصيل ابنائهم الدراسي، اقتصار التلاميذ المتفوقين على الدروس فقط، وعدم اهتمامهم بالأنشطة الإلكترونية الصفية،عدم توفر الإمكانات المادية والتجهيزات المختلفة داخل المدراس الحكومية لتنفيذ الأنشطة الإلكترونية، اذدحام الفصول بالتلاميذ لايثجع على اجراء الأنشطة الإلكترونية بطريقة جيدة، عدم اهتمام مديريرو المدارس بمتابعة تتفيذ المعلمين للأنشطة الإلكترونية، وكذلك عدم وجود الخبرة الكافية لدى معلمي مرحلة التعليم الأساسي باجر اء وتطبيق الأنشطة الإلكترونية الصفية،عدم وجود تدرييات للمعلمين على كيفية القيام بهذه الأنشطة، عدم ارتباط المنهج الدر اسي بالأنشطة الإلكترونية الصفية، ازدحام الجدول المدرسي بالحصص يؤثر سلبيا على أداء الأنشطة الإلكترونية لأنها تحتاح إلى وقت كبير في أدائها.

للإجابة على السؤال الثاني الذي ينص على التئ: ما هي التحديات التي تواجه تطبيق الأنثطة الصفية الإكترونية في مدارس التعليم

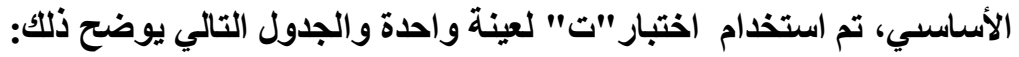

جدول(4): نتائج اختبار "ت" لفقرات البعد الثاني من استبيان تطبيق الأنشطة الصفية الإكترونية

\begin{tabular}{|c|c|c|c|c|}
\hline مستوى & "ت" & 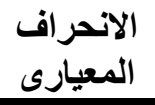 & المتوسط & الفقرة \\
\hline 0.01 & 10.78 & .597 & 1.48 & الاكترونية أنت المعلمين ليس لايهم القناعة الكافية بتطبيق الانشطة \\
\hline 0.01 & 11.67 & 0526 & 1.53 & اعفية الكترونية تغيير الانثطة التربوية المرتبطة بالمنهج الى انثطة \\
\hline 0.01 & 11.99 & .528 & 1.58 & 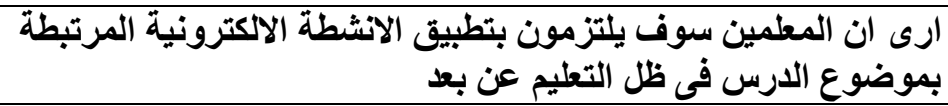 \\
\hline 0.01 & 13.061 & .608 & 1.67 & 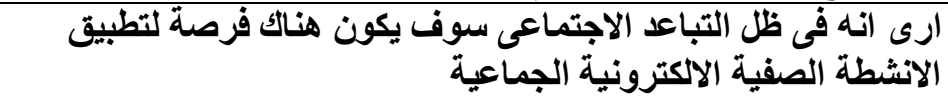 \\
\hline 0.01 & 9.594 & .677 & 1.48 &  \\
\hline 0.01 & 10.280 & .671 & 1.57 &  \\
\hline 0.01 & 11.308 & .636 & 1.59 & 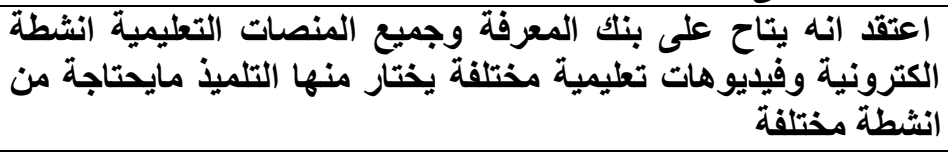 \\
\hline 0.01 & 9.181 & .689 & 1.54 & 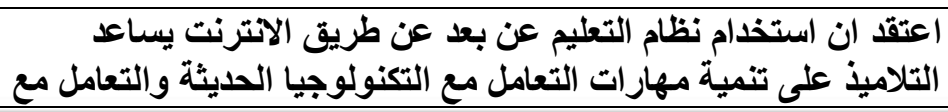 \\
\hline
\end{tabular}




\begin{tabular}{|c|c|c|c|c|}
\hline & & & & متطلبات العصر واستخدام الانثطة الاكترونية الصفية \\
\hline 0.01 & 11.884 & 660 & 1.59 & 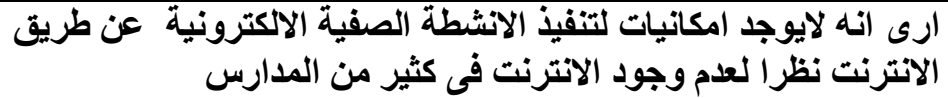 \\
\hline 0.01 & 11.686 & .593 & 1.49 & الاكترونية انليوجد خبرة لاى المعلمين فى تنفيذ الانشطة الصفية \\
\hline 0.01 & 11.260 & .741 & 1.57 & 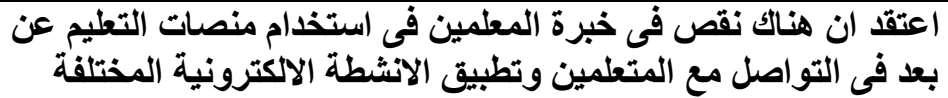 \\
\hline 0.01 & 10.687 & 617 & 2.59 & فى النفس ان استخدام الانشطة الاكترونية الصفية يساعد التلاميذ على الثقة \\
\hline 0.01 & 12.324 & .750 & 2.99 & البحث وان الابتكار والانفتاح الانشطة الاكتئرونية عميع الخبرات المختلفة يساعد التلاميذ على \\
\hline 0.01 & 14.357 & 689 & 2.58 & الدراسية ان تنفيذ الانشطة الاككترونية لا تتناسب مع جميع المواد \\
\hline
\end{tabular}

يتضح من الجدول السابق : إن جميع قيم "ت" دالة احصائيا كما أن جميع قيم المتوسطات والأوزان النسبية لجميع الفقرات لهذا البعد أكبر من (2) بدرجة طبيرة وقريبة من (13) أي أن هذه العبارات على درجة كبيرة من الاتفاق على وجود الكثير من التحديات التي تو اجهه تطبيق الأنشطة الصفية الإكترونية في مدارس التعليم الأساسي، ولقد اتفق مع هذه النتيجة بحث ويفر (weaver,1977) حيث هدف إلى التعرف على مكانة الأنشطة المدرسية اللاصفية ومكانتها في مناهج المدرسة، وعلى مدى الاهتمام بها وقد اقتصرت على الأنشطة الصفية فقط في مر احل التعليم المختلفة، وتم التوصل إلى أن هناك بعض المعوقات المرتبطة بالإمكانات المادية و البشرية وعدم أخذ الأنشطة في الحسبان عند تقويم التلاميذ، وقد فسرت الباحثة هذه النتيجة بأنه قد يرجع ذلك إلى مجموعة من التحديات الاقتصادية و الاكاديمية منها عدم توفر الانترنت في كثير من المدارس وضعف الانترنت وضعف الامكانيات المادية عدم وجود البنية التحتية لكثير من المدارس الأساسية من شبكات الانترنت، وكذلك عدم توفر أجزة كمبيونر في مدارس التعليم الأساسي، وضعف الإمكانات المادية لكثير من الأسر حيث لايستطيع كثير من التلاميذ تنفيذ هذه الأنشطة أو التواصل مع المعلم من خلال المنزل، ضعف قدرة المعلمين الأكاديمية فى تنفيذ الأنشطة الإلكترونية وفي تصميمها وفي إعدادها وعدم وجود تدريبات للمعلمين على هذه النوعيه من الأنشطة الإلكترونية.

\section{التوصيات}

• انشاء نظام رقمي متخصص في التعليم الإلكتروني للمراحل التعليم الأساسي كمرحلة أولى في التطبيق، الفكرة تكمن في توفير نظام در اسي إلكتروني يو ازي المادة المعطاة في المر احل الابتدائية ويغذيها ويدعمها بالأمتلة والمزيد من الثر ح و المحاكاة الواقعية بالاعتماد على توثيق كامل (فيديو ، فلاشات، ملفات صوتية وأمثلة ) للاروس المعطاة في المدارس لتكون مرجع دائم للطفل و الأهل. • توفر الميز انيات والامكانات المادية المطلوبة لانجاز الأنشطة الصفية الإكترونية بصورة مرضية. • وفير حو افز للطلبة والمعلمين و المديرين الذين يظهرون تفوقا وإبداعيا و إخلاصا في الأنشطة الصفية الإلكترونية. 
• ضرورة عمل تدريبات للمعلمين ومشرفي النشاط ومديرة المدارس على كيفية التعامل مع المنصات التعليمية وذلك لعرض الأنشطة الصفية الإلكترونية وكذلك مو اقع التواصل الاجتماعي. يجب استخدام نظام التعليم عن بعد جنبا إلى جنب مع النظام التقليدي. يجب دعم المدارس بشبكات الانترنت ودعم الانترنت لجميع الأفراد.

هجب توفير منصات تعليمية مختلفة عليها العديد من الأنشطة الإلكترونية المختلفة و المتنوعة ويكون سهلة فى الوصول إليها. توفير فيديو هات تعليمية ومصادر مختلفة لتنفيذ الأنشطة الصفية الإلكترونية على شبكة الانترنت. • يجب احتساب درجات الأنشطة الإلكترونية من ضمن درجات التلاميذ. • أن يقوم كل معلم برفع أنشطة إلكترونية مرتبطة بالدرس وتكون على شكل فبديو هات تعليمة على شبكات الانترنت وصفحات التو اصل الاجتماعي. 
قائمة المصار والمراجع:

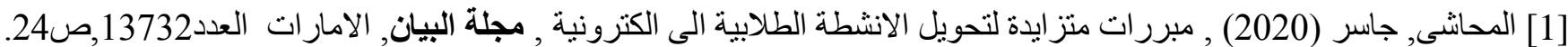

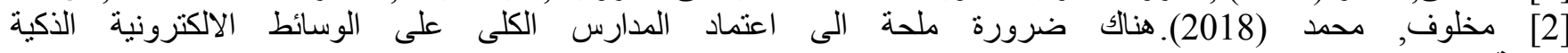
, مجلة البيان , الامارات ,العدد 13732-ص -20. [3] Woods, R., \& Baker, J.(2004). Interaction and Immediacy in Online Learning. The International Review of Research in Open and Distance Learning, 5(2). Retrieved dec. 10, 2012 Available At http://www.irrodl.org/index.php/irrodl/article/view/186/268

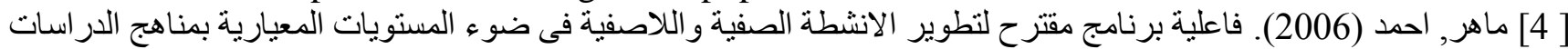
الاجتماعية فى تنمية مهار ات كتابة البحوث التاريخية لدى تلاميذ المرحلة الابتدائية , مجلة كلية التربية, جامعة بنها راكية , مصر .

[5] Salmon ,C.(2002).E -tivities ,The Key to active online Learning

[6]Palma,J\&Piteira,M.(2008).The activities value in e-learning. Available At http://eunis .dkjpapers/p23pfd.

[7] Creanor ,L\& penter (2004).E-Learning Guides : Activities for E-Learning Glasgow Caledonian University.

[8]- نبيل جاد عزمى (2008). تكنولوجيا التعليم الالكترونى , القاهرة, دار الفكر العربى .

[9] Alison ,B \& lirs ,V.(2002).Practical Tips for successful online Teaching, Teaching and Learning ,forum,Retrieved,oc11,2009. From .http://www.isn.curtin.edu.au/

[10] Aldrich ,C.(2005).Learning by doing :A comprehensive guide to simulation computer games and pedagogy in e-learning and other educational experiences .San Francisco ,CA: John Wiley \& Sons ,Inc.

$$
\begin{aligned}
& \text { [11 ] الكنعان, هدى محمد(2008). استخدام التعليم الالكترونى فى التدريس ,ورقة عمل مقدمة لملتقى التعليم الالكترونى الاول, فى }
\end{aligned}
$$

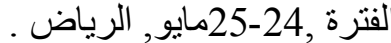

$$
\begin{aligned}
& \text { [12] الموسوى, على بن شرف (242020). الانشطة التعليمية وتطور ها باستخدام تقنيات التعليم و المعلومات ووسائطهما , مجلة بحوث }
\end{aligned}
$$

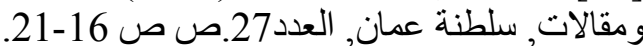

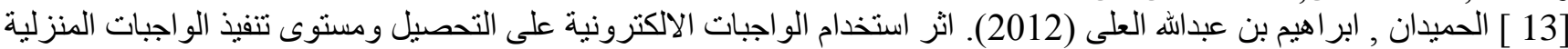





$$
\begin{aligned}
& \text { التفاعلى, الفصل الاكترونى التعاونى, الفصل الالكترونى التكاملى ) على تحصيل تلاميذ الصف الخامس الابتدائى لمادة الرياضيات , }
\end{aligned}
$$



[15] Kamel .S.(2000).The Web as a Learning Environment for Kids ,Distance a Learning Technologies ,Issues ,Trends and Opportunities, Edited by Linda Lau Group Publishing

[16] الطر ان, ايمان عبدالعاطى(2009). برنامج مقتر ح باستخدام ادوات التفاعل عبر شبكة الانترنت وتاثيره على طلاب كلية التربية فى وكي

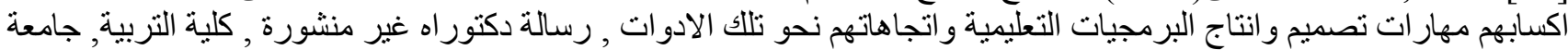

المنصورة.

[17] محمد نوفيق, زهو (2008) ـ تصور مقترح لتفعيل دور الانشطة المدرسية فى تنمية الابداع لدى تلاميذ الحلقة الاولى العي

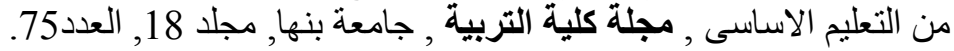



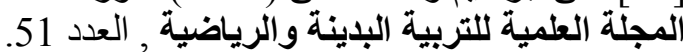

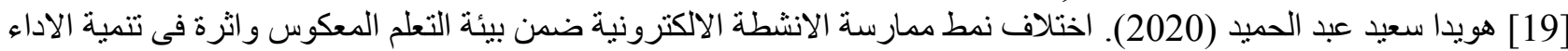

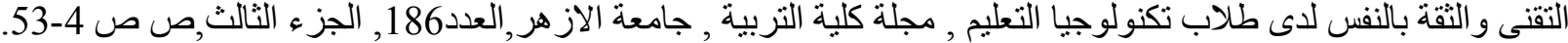

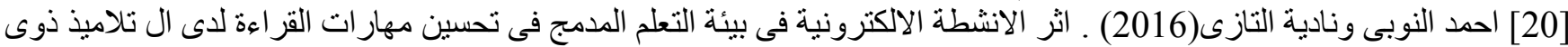



